\title{
Replication of the Wellcome Trust genome-wide association study on essential hypertension in a Korean population
}

\author{
Kyung-Won Hong ${ }^{1}$, Hyun-Seok Jin ${ }^{1}$, Yoon Shin $\mathrm{Cho}^{2}$, Jong-Young Lee ${ }^{2}$, Jong-Eun Lee ${ }^{3}$, Nam H Cho ${ }^{4}$, \\ Chol Shin ${ }^{5}$, Sang-Ho Lee ${ }^{6}$, Hun Kuk Park ${ }^{1}$ and Bermseok Oh ${ }^{1}$
}

Essential hypertension has a high rate of morbidity and mortality, primarily because of the associated complications. The Wellcome Trust Case Control Consortium recently conducted a genome-wide association study and identified six single nucleotide polymorphisms (SNPs) associated with essential hypertension. The Family Blood Pressure Program later investigated these six SNPs in an attempt to reproduce the aforementioned associations, but only one such association was found. However, this replication study did not include any Asian patients. Therefore, we investigated the association of the six SNPs with hypertension using 7551 unrelated individuals from the Korean Association REsource, a genome-wide association study based on epidemiological cohorts in Korea. We carried out a logistic regression analysis for hypertension status, and a linear regression analysis with systolic blood pressure and diastolic blood pressure as quantitative traits. Even though two SNPs, rs6997709 and rs7961152, were associated with systolic and diastolic blood pressures, respectively, in the quantitative trait association test, no associations were found using a case-control association test. The Korean Association REsource data consisted of two community cohorts (the Ansung and Ansan areas in Korea). The SNP rs7961152 was found to be associated with hypertension risk (OR (odds ratio) $=1.286, \mathrm{Cl}$ (confidence interval) $1.012-1.636, P=0.040$ ), but only in the Ansan cohort. This study as well as the Family Blood Pressure Program study show that because of the complexity of the genetic architecture, an association study with a larger sample population may be necessary to identify genetic variants that affect hypertension. Hypertension Research (2009) 32, 570-574; doi:10.1038/hr.2009.68; published online 8 May 2009

Keywords: SNP; KARE; FBPP

\section{INTRODUCTION}

Hypertension, defined as a systolic blood pressure (SBP) $\geqslant 140 \mathrm{~mm} \mathrm{Hg}$ and/or a diastolic blood pressure (DBP) $\geqslant 90 \mathrm{~mm} \mathrm{Hg}$, is an important risk factor for cardiovascular disease. ${ }^{1,2}$ Hypertension is a polygenic disease, with complex interactions of networked genes with environmental stimuli, and likely contributes to the development of metabolic syndrome, obesity and cardiovascular risk factors. ${ }^{3}$ To address the genetic risk factors for hypertension, many association studies have been conducted on the basis of casecontrol comparisons, as well as population-based analyses of both candidate genes and the entire genome. ${ }^{4-9}$ The first genome-wide association study was carried out by the Wellcome Trust Case Control Consortium (WTCCC), which enrolled 2000 hypertensive patients and 3000 normotensive controls. ${ }^{4}$ Although no single SNP (single nucleotide polymorphism) achieved genome-wide significance $\left(P<5 \times 10^{-7}\right)$, six variants were found to be highly associated with hypertension $\left(P<10^{-5}\right){ }^{4}$ These six SNPs were the focus of another study, the Family Blood Pressure Program (FBPP), in which 11433 individuals were recruited from hypertensive families. Although the FBPP did not reproduce the results of the WTCCC study, one (rs1937506) of the six SNPs was found to be associated with hypertension in Hispanic Americans and European Americans. ${ }^{5}$

To explore the genetic basis of prevalent, lifestyle-related complex diseases in Korea, we launched the KARE (Korean Association REsource) project in 2007. Participants $(n=10038)$ aged 40-69 years in the Ansung $(n=5018)$ and Ansan $(n=5020)$ community-based cohorts were genotyped using the Affymetrix SNP Array 5.0. As FBPP did not incorporate the Asian population, we analyzed the association of the six SNPs identified in the WTCCC study with SBP and DBP in 7551 unrelated individuals from the KARE project.

${ }^{1}$ Department of Biomedical Engineering, School of Medicine, Kyung Hee University, Seoul, Korea; ${ }^{2}$ Center for Genome Science, National Institute of Health, Seoul, Korea; ${ }^{3}$ DNA Link Inc., Seoul, Korea; ${ }^{4}$ Department of Preventive Medicine, Ajou University School of Medicine, Suwon, Korea; ${ }^{5}$ Department of Internal Medicine, Korea University Ansan Hospital, Gyeonggi-do, Korea and ${ }^{6}$ Department of Internal Medicine, Kyung Hee University East-West Neo Medical Center, Seoul, Korea Correspondence: Dr B Oh, Department of Biomedical Engineering, School of Medicine, Kyung Hee University, 1 Hoeki-dong, Dongdaemun-gu, Seoul 130-702, Korea. E-mail: ohbs@khu.ac.kr

Received 20 January 2009; revised 19 March 2009; accepted 30 March 2009; published online 8 May 2009 


\section{MATERIALS AND METHODS}

Subjects

Subjects were taken from two community-based cohorts, Ansung and Ansan, in South Korea. Both cohorts began in 2001 as a part of the Korean Genome Epidemiology Study. The initial populations of individuals, aged 40-69 years, from the rural community of Ansung and the urban community of Ansan were 5018 and 5020, respectively. Both cohorts are located in the KyungGi-Do province, which is near Seoul, Korea.

The basic characteristics and blood pressures of the patients are described in Table 1. For this study, blood pressure measurements were taken thrice in the supine position. Before the first measurement, participants had a $5 \mathrm{~min}$ rest, and then three measurements were taken at least 30 seconds apart. The average of these three measurements was used for this study. Hypertensive status was defined as a SBP $\geqslant 140 \mathrm{~mm} \mathrm{Hg}$ and/or a DBP $\geqslant 90 \mathrm{~mm} \mathrm{Hg}$.

\section{Genotyping and quality control}

Most DNA samples were isolated from the peripheral blood of participants and genotyped using the Affymetrix Genome-Wide Human SNP array 5.0. (Affymetrix Inc., Santa Clara, CA, USA). The accuracy of the genotyping was examined by Bayesian Robust Linear Modeling using the Mahalanobis Distance (BRLMM) Genotyping Algorithm. ${ }^{10}$ Samples with accuracies $<98 \%$, a high missing-genotype call rate $(\geqslant 4 \%)$, high heterozygosity $(>30 \%)$ or inconsistency in sex were excluded from subsequent analyses. Individuals with any kind of tumor were excluded. Related individuals whose estimated identity-by-state value was high $(>0.80)^{4}$ were also excluded. Finally, we used 8512 individuals, including 961 patients receiving antihypertensive therapy. Five of the six WTCCC SNPs were low in the missing-gene call rate $(<0.001)$, high in MAF $(>0.01)$ and not significantly deviated from the Hardy-Weinberg equilibrium $\left(P<1 \times 10^{-6}\right)$. One SNP ( rs 1937506) had a high missing-gene call rate (0.075), a high MAF and no deviation from HardyWeinberg equilibrium.

\section{Association tests}

Quantitative trait analysis. Patients receiving antihypertensive therapy were excluded in the first analysis. The final sample size for quantitative trait analysis study was 7551 individuals. Linear regression was used to analyze the SBP and DBP as quantitative traits, controlling for the patient's cohort, age, sex and body mass index (BMI).
Case-control analysis. We carried out two series of case-control analysis. The first set used the same sample of 7551 individuals used in quantitative trait analysis and the second set used 8512 subjects, including the patients of antihypertensive therapy. We defined untreated hypertensive patients as cases not including patients taking antihypertensive medication, and all hypertensive patients used in the second case-control analysis, as cases including 961 patients taking antihypertensive medication. In the second case-control study, we excluded the pre-hypertensive patients, defined as a SBP $<120 \mathrm{~mm} \mathrm{Hg}$ and a DBP $<80 \mathrm{~mm} \mathrm{Hg}$, from the control group. Logistic regression analysis was used for the hypertensive cases and normotensive controls, controlling for cohort, age, sex and BMI as covariates.

Statistical analysis. Statistical analyses were carried out using SPSS (version 15.0; SPSS Inc., Chicago, IL, USA). All tests were based on an additive model and $P$-values were not adjusted for multiple tests.

\section{RESULTS}

\section{KARE hypertension data set and allele frequencies}

We analyzed 7551 unrelated individuals in KARE, 3470 subjects from the Ansung cohort and 4081 subjects from the Ansan cohort. The basic characteristics of the subjects are described in Table 1. The regional differences were computed using a $\chi^{2}$-test for both sex and hypertensive ratio, whereas an independent student's $t$-test was used for age, BMI, SBP and DBP. All variables were significantly different between the Ansung and Ansan cohorts, as reported earlier by Kim et al. ${ }^{11}$

The minor allele frequencies of the six SNPs in the KARE data were compared with the minor allele frequencies from the WTCCC data and dbSNP (The Single Nucleotide Polymorphism database) data. As shown in Table 2, the allele frequencies in the KARE data were similar to those in the HCB (Han Chinese in Beijing) and JPT (Japanese in Tokyo), but were different from those of the WTCCC and CEU (Caucasians in Europe).

\section{Association tests}

Quantitative trait analysis. The quantitative trait analyses for SBP and DBP were carried out using linear regression analysis, controlling

Table 1 Basic characteristics of study subjects

\begin{tabular}{|c|c|c|c|c|c|c|}
\hline & \multirow[b]{2}{*}{ Total } & \multicolumn{2}{|c|}{ Ansung } & \multicolumn{2}{|c|}{ Ansan } & \multirow{2}{*}{$\begin{array}{c}\text { P-value } \\
\text { Ansung and Ansan }\end{array}$} \\
\hline & & Men & Women & Men & Women & \\
\hline \multicolumn{7}{|l|}{ Quantitative trait analysis } \\
\hline Number of individuals (\%) & 7551 & \multicolumn{2}{|c|}{$3470(46)$} & \multicolumn{2}{|c|}{$4081(54)$} & \\
\hline Gender (men (\%) /women (\%)) & $3747(50) / 3804(50)$ & \multicolumn{2}{|c|}{$1584(46) / 1886(54)$} & \multicolumn{2}{|c|}{$2163(53) / 1918(47)$} & $<0.001$ \\
\hline Age (mean $(M)$ year \pm standard deviation (s.d.)) & $51.44 \pm 8.79$ & $55.53 \pm 8.67$ & $54.61 \pm 8.91$ & $48.16 \pm 7.25$ & $48.63 \pm 7.80$ & $<0.001$ \\
\hline Body mass index (BMI) $\left(M \mathrm{~kg} \mathrm{~m}^{-2} \pm \mathrm{s} . \mathrm{d}.\right)$ & $24.42 \pm 3.08$ & $23.59 \pm 3.07$ & $24.74 \pm 3.27$ & $24.55 \pm 2.71$ & $24.62 \pm 3.17$ & $<0.001$ \\
\hline Systolic blood pressure (SBP) ( $M \mathrm{~mm} \mathrm{Hg} \pm$ s.d.) & $115.65 \pm 17.25$ & $120.93 \pm 16.42$ & $120.07 \pm 17.81$ & $113.26 \pm 15.20$ & $109.66 \pm 17.08$ & $<0.001$ \\
\hline Diastolic blood pressure (DBP) ( $M \mathrm{~mm} \mathrm{Hg} \pm$ s.d.) & $74.21 \pm 11.27$ & $77.46 \pm 9.67$ & $75.85 \pm 10.23$ & $74.52 \pm 11.71$ & $69.56 \pm 11.40$ & $<0.001$ \\
\hline \multicolumn{7}{|l|}{ Case-Control analysis } \\
\hline \multicolumn{7}{|l|}{ For untreated hypertensive patients } \\
\hline Control (SBP <140 mm Hg and DBP <80 mm Hg) & 6667 & \multicolumn{2}{|c|}{2937} & \multicolumn{2}{|c|}{3730} & \\
\hline Cases (SBP $\geqslant 140 \mathrm{~mm} \mathrm{Hg}$ and/or DBP $\geqslant 90 \mathrm{~mm} \mathrm{Hg}$ ) & 884 & \multicolumn{2}{|c|}{533} & \multicolumn{2}{|c|}{351} & \\
\hline \multicolumn{7}{|l|}{ For all hypertensive patients } \\
\hline Control (SBP $<120 \mathrm{~mm} \mathrm{Hg}$ and DBP $<80 \mathrm{~mm} \mathrm{Hg}$ ) & 4451 & \multicolumn{2}{|c|}{1700} & \multicolumn{2}{|c|}{2751} & \\
\hline Patients with hypertension therapy & 961 & \multicolumn{2}{|c|}{579} & \multicolumn{2}{|c|}{382} & \\
\hline $\begin{array}{l}\text { Cases (untreated hypertensive patients and } \\
\text { those undergoing hypertension therapy) }\end{array}$ & 1845 & \multicolumn{2}{|c|}{1112} & \multicolumn{2}{|c|}{733} & \\
\hline
\end{tabular}

a $P$-values were obtained using the $\chi^{2}$-test for the sex and hypertensive variables, and by independent Student's $t$-test for other variables. 


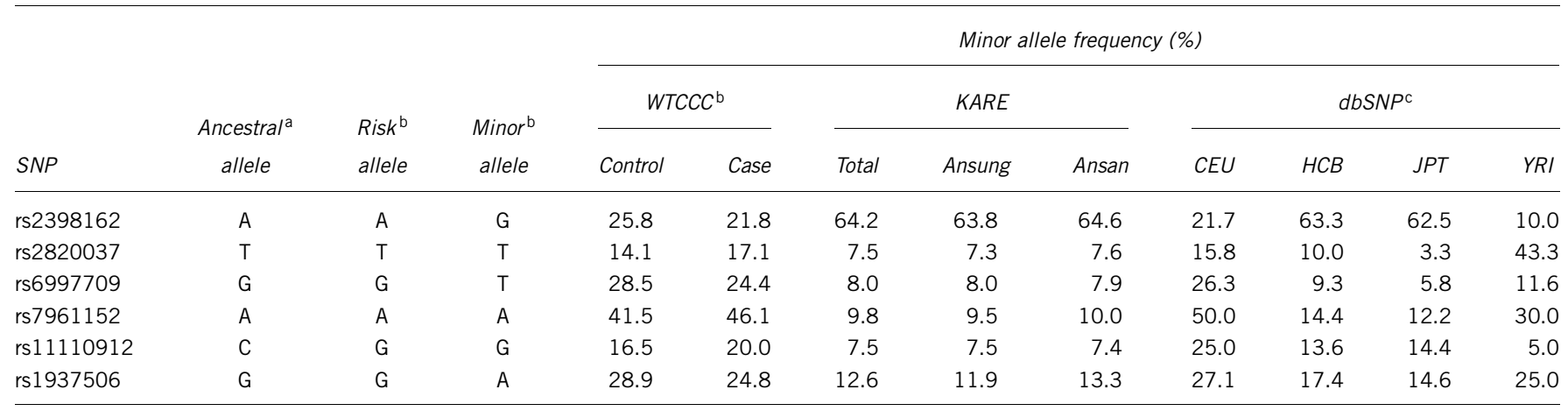

Abbreviations: CEU, European cohort; dbSNP, The Single Nucleotide Polymorphism database; HCB, Han-Chinese cohort; JPT, Japanese cohort; KARE, Korean Association Resource; SNP, single nucleotide polymorphism; WTCCC, The Wellcome Trust Case Control Consortium; YRI, Sub-Saharan African cohort.

aChimpanzee homolog sequence obtained from GenBank database (http://www.ncbi.nlm.nih.gov/).

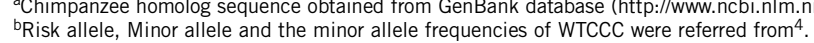

cdbSNP minor allele frequencies were obtained from the NCBI dbSNP database (http://www.ncbi.nlm.nih.gov/sites/entrez?db =snp\&cmd=search\&term=).

Table 3 Linear regression analysis for systolic and diastolic blood pressures, controlling for cohort, age, sex and BMI

\begin{tabular}{|c|c|c|c|c|c|c|}
\hline \multirow[b]{2}{*}{ Patients } & \multirow[b]{2}{*}{$S N P$} & \multirow[b]{2}{*}{ Reference allele } & \multicolumn{2}{|c|}{ Systolic blood pressure } & \multicolumn{2}{|c|}{ Diastolic blood pressure } \\
\hline & & & $\mathrm{P}$-value & B & P-value & $\mathrm{B}$ \\
\hline \multicolumn{7}{|l|}{ All } \\
\hline \multirow{6}{*}{$(n=7751)$} & rs2398162 & $A$ & 0.969 & 0.010 & 0.479 & 0.128 \\
\hline & rs2820037 & $\mathrm{T}$ & 0.392 & -0.409 & 0.304 & -0.333 \\
\hline & rs6997709 & G & $\underline{0.059}$ & -0.881 & $\underline{0.009}$ & -0.827 \\
\hline & rs7961152 & A & $\overline{0.008}$ & 1.119 & $\overline{0.089}$ & 0.489 \\
\hline & rs11110912 & C & 0.832 & -0.102 & 0.705 & -0.123 \\
\hline & rs1937506 & G & 0.590 & -0.295 & 0.169 & -0.418 \\
\hline \multicolumn{7}{|l|}{ Ansung } \\
\hline \multirow[t]{6}{*}{$(n=3470)$} & rs2398162 & $A$ & 0.138 & 0.604 & 0.146 & 0.352 \\
\hline & rs2820037 & $\mathrm{T}$ & 0.799 & -0.186 & 0.534 & -0.269 \\
\hline & rs6997709 & G & 0.693 & -0.282 & 0.500 & -0.287 \\
\hline & rs7961152 & $A$ & 0.138 & 0.979 & 0.376 & 0.348 \\
\hline & rs11110912 & C & 0.190 & -0.953 & 0.128 & -0.658 \\
\hline & rs1937506 & $\mathrm{G}$ & 0.789 & -0.183 & 0.956 & -0.023 \\
\hline \multicolumn{7}{|l|}{ Ansan } \\
\hline \multirow[t]{6}{*}{$(n=4081)$} & rs2398162 & $A$ & 0.211 & -0.441 & 0.988 & -0.004 \\
\hline & rs2820037 & $\mathrm{T}$ & 0.312 & -0.636 & 0.367 & -0.424 \\
\hline & rs6997709 & $\mathrm{G}$ & $\underline{0.026}$ & -1.364 & $\underline{0.006}$ & -1.266 \\
\hline & rs7961152 & $A$ & $\overline{0.018}$ & 1.310 & $\overline{0.103}$ & 0.672 \\
\hline & rs11110912 & C & $\overline{0.300}$ & 0.660 & 0.453 & 0.357 \\
\hline & rs1937506 & $G$ & 0.530 & -0.368 & 0.100 & -0.722 \\
\hline
\end{tabular}

Abbreviations: BMI, body mass index; SNP, single nucleotide polymorphism.

Underlined values indicate the significant results based on $P<0.1$.

for cohort, sex, age and BMI (Table 3). As shown by these analyses, two SNPs, rs6997709 and rs7961152, were found to be significantly associated with blood pressure. The SNP rs6997709 trended toward an association with SBP $(P=0.059, B=-0.881)$ and was significantly associated with DBP $(P=0.009, B=-0.827)$ in the direction of decrease. The SNP rs7961152 was significantly associated with SBP $(P=0.008, B=1.119)$ and trended toward association with DBP $(P=0.089, B=0.489)$ in the direction of increase. The quantitative trait analysis of each individual cohort showed similar results in the Ansan cohort, but not in the Ansung cohort (Table 3). In the Ansan cohort, the SNP rs6997709 was significantly associated with both SBP $(P=0.026, B=-1.364)$ and DBP $(P=0.006, B=-1.266)$, and the SNP rs7961152 was associated with both $\operatorname{SBP}(P=0.018, B=1.310)$ and DBP $(P=0.103, B=0.672)$.
Case-control analysis. For the two case-control study sets, logistic regression analysis was used (Table 4). We controlled for confounding factors, such as cohort, sex, age and BMI. When looking at all patients, no significant associations were found between the untreated hypertensive patients and any of the six SNPs. Given that the basic characteristics in blood pressures were significantly different between the Ansung and Ansan cohorts, we also tested each cohort separately. The SNP rs7961152 was found to have a positive association with hypertensive status in the Ansan cohort ( $\mathrm{OR}=1.286$, CI 1.012-1.636, $P=0.040$ ), but no such association in the Ansung cohort was found ( $\mathrm{OR}=1.048$, CI $0.834-1.317, P=0.688$ ).

In the second analysis, we added patients on antihypertensive therapy to the cases, and excluded the pre-hypertensive patients from the control group. One SNP, rs2820037, revealed a significant 
Table 4 Logistic regression analysis for untreated hypertensive patients and all hypertensive patients controlling for cohort, age, sex and BMI

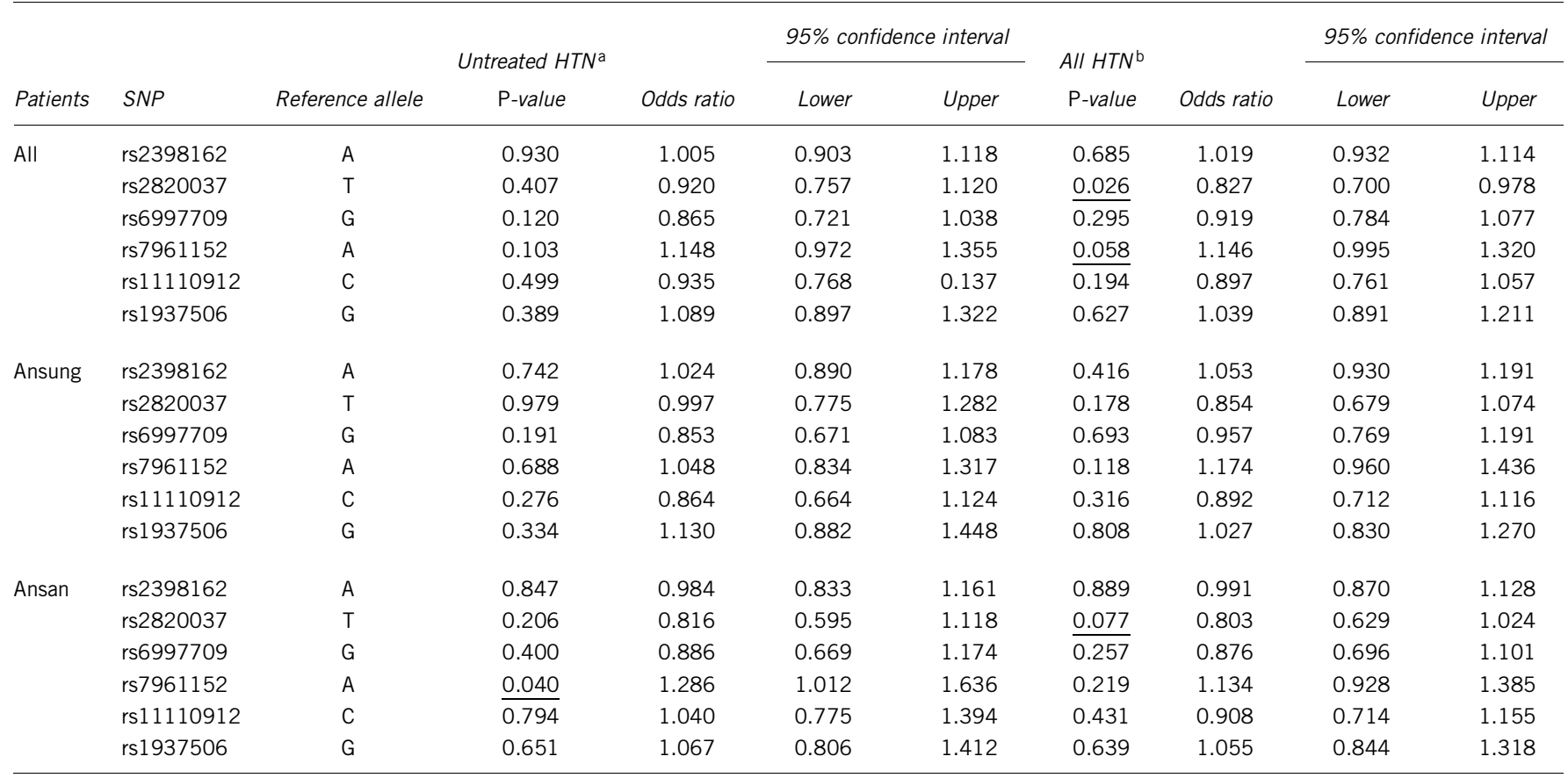

Abbreviations: BMI, body mass index; HTN, hypertensive patients; SNP, single nucleotide polymorphism.

Underlined values indicate the significant results based on $P<0.1$.

${ }^{a}$ Cases were patients with SBP $\geqslant 140 \mathrm{~mm} \mathrm{Hg}$ and/or DBP $\geqslant 90 \mathrm{~mm} \mathrm{Hg}$, and controls were patients with SBP $<140 \mathrm{~mm} \mathrm{Hg}$ and DBP $<80 \mathrm{~mm} \mathrm{Hg}$.

${ }^{b}$ Cases included untreated HTN and patients of hypertension therapy, and controls were patients with SBP $<120 \mathrm{~mm} \mathrm{Hg}$ and DBP $<80 \mathrm{~mm} \mathrm{Hg}$ excluding the pre-hypertensive status.

Table 5 Comparison of the association status of each SNP with hypertension in the KARE, WTCCC and FBPP studies

\begin{tabular}{|c|c|c|c|c|c|c|c|c|c|}
\hline \multirow[b]{2}{*}{ Patients } & \multirow[b]{2}{*}{ SNP } & \multirow[b]{2}{*}{ Reference allele } & \multicolumn{3}{|c|}{$K A R E$} & \multirow[b]{2}{*}{ WTCCC } & \multicolumn{3}{|c|}{$F B P P$} \\
\hline & & & All & Ansung & Ansan & & African Americans & European Americans & Hispanic Americans \\
\hline Control & & & 6867 & 2937 & 3730 & 3000 & 1833 & 2101 & 1746 \\
\hline \multirow[t]{7}{*}{ Case } & & & 884 & 533 & 351 & 2000 & 3135 & 2878 & 900 \\
\hline & rs2398162 & $A$ & 0.930 & 0.742 & 0.847 & $5.67 \times 10^{-5}$ & 0.400 & 0.640 & 0.068 \\
\hline & rs2820037 & $\mathrm{T}$ & 0.407 & 0.979 & 0.206 & $7.66 \times 10^{-7}$ & 0.180 & 0.830 & 0.300 \\
\hline & rs6997709 & $\mathrm{G}$ & 0.120 & 0.191 & 0.400 & $4.36 \times 10^{-5}$ & 0.740 & 0.670 & 0.320 \\
\hline & rs7961152 & $A$ & 0.103 & 0.688 & 0.040 & $3.03 \times 10^{-5}$ & 0.340 & 0.440 & 0.060 \\
\hline & rs11110912 & C & 0.499 & 0.276 & 0.794 & $1.94 \times 10^{-5}$ & 0.660 & 0.910 & 0.420 \\
\hline & rs1937506 & $\mathrm{G}$ & 0.389 & 0.334 & 0.651 & $4.53 \times 10^{-6}$ & 0.550 & 0.600 & 0.060 \\
\hline
\end{tabular}

Abbreviations: FBPP, Family Blood Pressure Program; KARE, Korean Association REsource from this study; WTCCC, Welcome Trust Case Control Consortium; SNP, single nucleotide polymorphism.

association with hypertension when considering all patients as a group (OR=0.827, CI $0.700-0.978, P=0.026$ ), and the $P$-value of rs7961152 was improved from 0.103 to 0.058 . When the Ansung and Ansan cohorts were tested separately, the SNP rs2820037 was not found to be associated with hypertension.

\section{DISCUSSION}

We carried out a replication study of the six SNPs highlighted in the WTCCC hypertension study. A quantitative trait study indicated the association of two SNPs (rs6997709 and rs7961152) with both SBP and DBP. Even though the rs7961152 was not found to be significantly associated with hypertension in a test of all patients, the SNP showed a trend of association with $P$-value of 0.103 , and was significantly associated with hypertension in the Ansan cohort $(P=0.040)$. The SNP rs7961152 also presented a tendency to associate with hypertension in the second case-control analysis that included patients taking antihypertensive medication $(P=0.058)$.

The SNP rs7961152 is an intronic SNP located on the branched chain aminotransferase 1 (BACT1) gene locus. This enzyme catalyzes the reversible transamination of branched-chain alpha-keto acids to branched-chain L-amino acids, which are essential for cell growth. ${ }^{12}$ No functional relationship between BCAT1 and hypertension has yet been reported. On the other hand, SNP rs6997709 and SNP rs2820037 are intergenic and not linked to any genes.

Table 5 shows the significance levels of the six SNPs from the KARE, WTCCC and FBPP studies. The SNP rs7961152, which was statistically significant in this study, showed a trend toward association in Hispanic Americans from the FBPP study $(P=0.060)$. On the other hand, the association of SNP rs1937506 with hypertension, which the FBPP study showed by a quantitative trait analysis in Hispanic and European Americans, was not significant according 
to linear regression analysis in the KARE study. However, the SNP rs1937506 had a high genotype-missing rate (0.075) in the KARE data, and its association may need further analysis using an improved genotyping method. Interestingly, the most significant SNP in the WTCCC study, rs2820037, showed significant association in our second case-control analysis, which incorporated antihypertension medication patients (Table 4). However, the SNP rs2820037 did not show any trend in the FBPP study. The significance of any SNPs in this study did not hold after Bonferroni correction for multiple testing.

As the prevalence, age of onset, severity and complications of hypertension are not similar among different ethnic groups, it is reasonable to assume that the contributions of the dissimilar gene sets differentially affect the development of hypertension in diverse populations. ${ }^{3}$ Moreover, the inconsistent results across the three studies suggest that the complex nature of the genetic architecture affects the development of essential hypertension. Therefore, to identify the genetic risk factors for hypertension, a more comprehensive study, with a larger sample population, may be necessary.

\section{ACKNOWLEDGEMENTS}

This work was supported by an extramural grant from the National Institute of Health, Korea (2008-E00355-00).

1 Himmelmann A, Hedner T, Hansson L, O'Donnell CJ, Levy D. Isolated systolic hypertension: an important cardiovascular risk factor. Blood Press 1998; 7: 197-207.
2 Noto D, Cefalu AB, Barbagallo CM, Sapienza M, Cavera G, Nardi I, Pagano M, Vivona N, Notarbartolo A, Averna MR. Hypertension and diabetes mellitus are associated with cardiovascular events in the elderly without cardiovascular disease. Results of a 15-year follow-up in a Mediterranean population. Nutr Metab Cardiovasc Dis 2008; doi:10.1016/j.numecd.2008.03.002.

3 Saavedra JM. Studies on genes and hypertension: a daunting task. J Hypertens 2005; 23: 929-932.

4 Wellcome Trust Case Control Consortium. Genome-wide association study of 14,000 cases of seven common diseases and 3000 shared controls. Nature 2007; 447: 661-678.

5 Ehret GB, Morrison AC, O'Connor AA, Grove ML, Baird L, Schwander K, Weder A, Cooper RS, Rao DC, Hunt SC, Boerwinkle E, Chakravarti A. Replication of the Wellcome Trust genome-wide association study of essential hypertension: the Family Blood Pressure Program. Eur J Hum Genet 2008; 16: 1507-1511.

6 Padmanabhan S, Melander O, Hastie C, Menni C, Delles C, Connell JM, Dominiczak AF. Hypertension and genome-wide association studies: combining high fidelity phenotyping and hypercontrols. J Hypertens 2008; 26: 1275-1281.

7 Lalouel JM, Rohrwasser A. Power and replication in case-control studies. Am J Hypertens 2002; 15: 201-205.

8 Cha SH, Kim HT, Jang Y, Park S, Kim JJ, Song MY, Park JH, Ryu HJ, Park HY, Yoon SJ, Kimm K, Lee JK, Oh B. Association of alpha-adducin Gly460Trp polymorphism with coronary artery disease in a Korean population. $J$ Hypertens 2007; 25: 2413-2420.

9 Iwai N, Tago N, Yasui N, Kokubo Y, Inamoto N, Tomoike H, Shioji K. Genetic analysis of 22 candidate genes for hypertension in the Japanese population. J Hypertens 2004; 22: 1119-1126.

10 Rabbee N, Speed TP. A genotype calling algorithm for affymetrix SNP arrays. Bioinformatics 2006; 22: 7-12.

11 Kim BG, Park JT, Ahn Y, Kimm K, Shin C. Geographical difference in the prevalence of isolated systolic hypertension in middle-aged men and women in Korea: the Korean Health and Genome Study. J Hum Hypertens 2005; 19: 877-883.

12 Sakamoto K, Yamasaki Y, Kaneto H, Fujitani Y, Matsuoka T, Yoshioka R, Tagawa T, Matsuhisa M, Kajimoto $Y$, Hori M. Identification of oxidative stress-regulated genes in rat aortic smooth muscle cells by suppression subtractive hybridization. FEBS Lett 1999; 461: 47-51. 